\title{
Threshold Secret Image Sharing
}

\author{
Teng Guo ${ }^{1,2}$, Feng $\mathrm{Liu}^{1}$, ChuanKun $\mathrm{Wu}^{1}$, ChingNung Yang ${ }^{3}$, Wen Wang $^{1,2}$, \\ and YaWei Ren ${ }^{1,2,4}$
}

1 State Key Laboratory of Information Security, Institute of Information Engineering,

Chinese Academy of Sciences, Beijing 100093, China

2 University of Chinese Academy of Sciences, Beijing 100190, China

3 Department of Computer Science and Information Engineering,

National Dong Hwa University, Hualien 974, Taiwan

4 School of Information Management,

Beijing Information Science and Technology University, Beijing 100192, China

\{guoteng, liufeng, ckwu, wangwen\}@iie.ac.cn, cnyang@mail.ndhu.edu.tw

\begin{abstract}
A $(k, n)$ threshold secret image sharing scheme, abbreviated as $(k, n)$-TSISS, splits a secret image into $n$ shadow images in such a way that any $k$ shadow images can be used to reconstruct the secret image exactly. In 2002, for $(k, n)$-TSISS, Thien and Lin reduced the size of each shadow image to $\frac{1}{k}$ of the original secret image. Their main technique is by adopting all coefficients of a $(k-1)$-degree polynomial to embed the secret pixels. This benefit of small shadow size has drawn many researcher's attention and their technique has been extensively used in the following studies. In this paper, we first show that this technique is neither information theoretic secure nor computational secure. Furthermore, we point out the security defect of previous $(k, n)$-TSISSs for sharing textual images, and then fix up this security defect by adding an AES encryption process. At last, we prove that this new $(k, n)$-TSISS is computational secure.
\end{abstract}

Keywords: Secret image sharing, Security defect, Computational secure.

\section{Introduction}

Secret image sharing has drawn considerable attention in recent years $[2,5,12$, 14, 18 21, 25 27]. A $(k, n)$ threshold secret image sharing scheme, abbreviated as $(k, n)$-TSISS, encrypts a secret image into $n$ shadow images (also referred to be shadows) in such a way that any $k$ shadows can be used to reconstruct the secret image exactly, but any less than $k$ shadows should provide no information about the secret image. The secret pixel can be hidden in the constant term of a $(k-1)$ degree polynomial using Shamir's $(k, n)$ secret sharing scheme, abbreviated as Shamir's $(k, n)$-SSS [17], and the secret image can be perfectly reconstructed from any $k$ shadows by Lagrange's interpolation. In such a case, each shadow is the same size as the secret image. For example, to encrypt a $10 G B$ satellite image by a $(5,10)$-TSISS, we get 10 shadows, each with size $10 G B$; and to

S. Qing et al. (Eds.): ICICS 2013, LNCS 8233, pp. 404-412, 2013.

(C) Springer International Publishing Switzerland 2013 
reconstruct the $10 G B$ satellite image, we have to collect 5 shadows, which sum up to $50 G B$. The larger the amount of information grows, the severer the above problem suffers from. To solve this large shadow size problem in secret image sharing, Thien and Lin [18] embed the secret pixels in all coefficients of a $(k-1)$ degree polynomial and reduce the shadow size to $\frac{1}{k}$ of the secret image. This variant use of Shamir's $(k, n)$-SSS is denoted as $(k, n)$-VSSS in this paper. Since the smaller shadow size makes the transmission and storage more convenient, the $(k, n)$-VSSS has drawn many attentions in the following studies 2, 3, 5, 12, 14, 18 22, 24 28] of TSISS ever since. Initially, Thien and Lin [18] adopt $G F(251)$ as the coefficient field, and the pixel's gray-level degrees has to be modified to less than 251. Therefore, Thien and Lin's scheme is in fact a lossy secret image sharing scheme, in which the reconstructed secret image may be distorted slightly in gray-level. In 2007, Yang et al. 23] adopt $G F\left(2^{8}\right)$ as the coefficient field, avoiding the losses in gray-level. Recently, $(k, n)$-TSISS has been combined with steganography and authentication [2, 3, [5, 24, 27], which divides a secret image into several shadows and embeds the produced shadows in the cover images to form the stego images, which can be transmitted to authorized recipients without causing suspicion. In addition, these schemes also have some authentication mechanisms to verify the integrity of the stego images, so that the secret image can be reconstructed correctly. $(k, n)$-TSISS has also been combined with visual cryptography [1, $8-11,15,16]$, which provides a two-in-one $(k, n)$ TSISS [14, 25] with two decoding options: the first option is stacking shadows to see a vague reconstructed image like visual cryptography; and the second option is to perfectly reconstruct the original gray-level secret image by Lagrange's interpolation.

However, there is no free lunch. The $(k, n)$-VSSS is no longer information theoretic secure, and to the best of our knowledge, no research has conjectured that the inverting of a $k-1$ degree polynomial $f(x)$ from less than or equal to $k-1$ shadows is computational infeasible. From this viewpoint, all of the above mentioned studies of $(k, n)$-TSISS provide neither of the two currently well-known security guarantees: 1, information theoretic security (also known as perfect secrecy), which is based on Shannon's information theory, e.g. the one-time pad, Shamir's secret sharing scheme, visual cryptography; 2, computational security, which is based on computational hardness assumptions, e.g. $R S A, A E S, D E S$. Motivated by the above observation, we in fact find the security defect of previous $(k, n)$-TSISSs for sharing textual images, in which the secret can be perceived from any single shadow. Please refer to Section 3 .

To avoid the above security defect, we suggest to add an $A E S$ encryption process before the sharing process to form a computational secure $(k, n)$-TSISS, which is denoted by $(k, n)$-CSTSISS. Then we prove it is computational secure by giving a construction that transforms any efficient attack of the $(k, n)$-CSTSISS to an efficient attack of $A E S$. In addition to theoretic analysis, experimental results are given to show feasibility of the proposed scheme. Compared to previous $(k, n)$-TSISSs, the proposed $(k, n)$-CSTSISS needs $256 n$ bits more storage space in overall, and more time for the $A E S$ encryption and decryption processes. 
This paper is organized as follows. In Section 2, we give some preliminaries of TSISS. In Section 3 , we point out the security defect of the previous $(k, n)$ TSISSs. In Section 4, we propose a computational secure $(k, n)$-TSISS. The paper is concluded in Section 5 .

\section{Preliminaries}

In this section, we first give some basic knowledge of Shamir's secret sharing and its variant version that is commonly used in studies of TSISS, and then analyze their security properties sequentially.

Suppose the secret we are going to share is in some finite field, e.g. prime fields $G F(p)$ or prime power fields $G F\left(2^{n}\right)$. For simplicity, we will take $G F(p)$ for example to illustrate the sharing process in the following. This will not cause any limitations, for the underlying principle is the same except that the operations in $G F\left(2^{n}\right)$ are modular of some irreducible polynomial of degree $n$, while those of $G F(p)$ are modular of prime number $p$.

In Shamir's $(k, n)$-SSS, to divide the secret $S \in G F(p)$ into $n$ shadows $S_{i} \in$ $G F(p)(1 \leq i \leq n)$, we first pick up $k-1$ random numbers $a_{1}, a_{2}, \ldots, a_{k-1}$ from $G F(p)$ and form a $k-1$ degree polynomial $f(x)=a_{0}+a_{1} x+a_{2} x^{2}+a_{3} x^{3}+\ldots+$ $a_{k-1} x^{k-1}$ with $a_{0}=S$. Then we evaluate each shadow by $S_{i}=f(i)(1 \leq i \leq n)$. From any $k$-subset of these $S_{i}$ values, we can reconstruct $f(x)$ by Lagrange's interpolation and compute the secret by $S=f(0)$. However, from any $(k-1)-$ subset of these $S_{i}$ values, we get no information about $S$. Detailed analysis can be found in [17].

In the $(k, n)$-VSSS, which is widely used in the studies of $(k, n)$-TSISS, to divide the secret $D=\left(D_{0}, D_{1}, \ldots, D_{k-1}\right)$ with $D_{0}, D_{1}, \ldots, D_{k-1} \in G F(p)$ into $n$ shadows $S_{i} \in G F(p)(1 \leq i \leq n)$, we first form a $k-1$ degree polynomial $f(x)=D_{0}+D_{1} x+D_{2} x^{2}+D_{3} x^{3}+\ldots+D_{k-1} x^{k-1}$. Then we evaluate each shadow by $S_{i}=f(i)(1 \leq i \leq n)$. From any $k$-subset of these $S_{i}$ values, we can reconstruct $f(x)$ by Lagrange's interpolation and obtain all the coefficients $\left(D_{0}, D_{1}, \ldots, D_{k-1}\right)=D$. Detailed analysis of the information leakage can be found in the proof of Theorem 2 .

To analyze the information leakage of the $(k, n)$-VSSS from less than $k$ shadows, we have to assume a probability distribution on the secret. For the simplicity of analysis and consistency with our proposed scheme, we assume that the secret is uniformly distributed in its space. For some knowledge of information theory, one can refer to [4]. Here we only give some necessary backgrounds. Entropy is a measure of the uncertainty associated with a random variable. Suppose $X$ is a random variable, its entropy is defined by $H(X)=\sum_{x \in X} p(x) \log \frac{1}{p(x)} \frac{1}{1}$. The amount of randomness in random variable $X$ given that you know the value of random variable $Y$ is defined by $H(X \mid Y)=\sum_{x \in X, y \in Y} p(x, y) \log \frac{p(y)}{p(x, y)}$, which is

\footnotetext{
${ }^{1}$ In this paper, the base of logarithm is 2 .
} 
also known as the conditional entropy of $X$ and $Y$. Briefly speaking, a $(k, n)$-SSS is information theoretic secure if any $k-1$ shadows provide no information about the secret. Formally, this notion is given as follows.

Definition 1 (Information theoretic secure). In a ( $k, n)-S S S$, suppose the secret is distributed according to random variable $S$ and the $n$ shadows are distributed according to random variables $S_{1}, S_{2}, \ldots, S_{n}$. The $(k, n)$-SSS is information theoretic secure if $H(S)=H\left(S \mid S_{i_{1}}, \ldots, S_{i_{k-1}}\right)$ holds for any $k-1$ shadows $S_{i_{1}}, \ldots, S_{i_{k-1}}$.

Theorem 1 ([17]). Shamir's $(k, n)$-SSS is information theoretic secure.

Theorem 2. In the $(k, n)$-VSSS, there is only $\frac{1}{k}$ fraction of the uncertainty of the secret left, given the knowledge of any $k-1$ shadows.

Due to page limit, the proof of Theorem 2 is omitted in this version. If you are interested in details of the proof, you can refer to the full version on eprint.

In the following, we present the common subprogram that all the above studies of $(k, n)$-TSISS share formally as Construction 1 .

\section{Construction 1.}

Input: $A$ secret image $S$.

Output: $n$ shadows $S_{1}, S_{2}, \ldots, S_{n}$.

Step 1. Adopt all coefficients of a $k-1$ degree polynomial $f(x)$ to embed the secret pixels of $S$. Each time we share $k$ successive pixels, say $p_{1}, p_{2}, \ldots, p_{k}$. Then fix $f(x)=p_{1}+p_{2} x+p_{3} x^{2}+\ldots+p_{k} x^{k-1}$. The pixel value for each shadow is calculated as $q_{i}=f(i)$ for $i=1,2, \ldots, n$. Repeat the above process until all pixels of $S$ have been shared. The shadows are denoted as $S_{1}, S_{2}, \cdots, S_{n}$. Step 2. Participant $i$ is distributed $S_{i}$ for $i=1,2, \ldots, n$.

Remark: In Step 1., we use the $(k, n)$-VSSS, whose security is not guaranteed. This may cause hidden security risk to Construction 1 Indeed we have found its security defect for sharing textual images, please refer to Section 3 .

\section{The Security Defect of Construction 1}

In this section, we present some experimental results to illustrate the security defect of Construction 1, while concrete theoretical analysis of the experiment is given in Appendix of the full version on eprint.

Here we only give a general idea of the cause of the security defect. Since the sharing process (Step 1.) of Construction 1 is deterministic, the same combination of $k$ secret pixels will always contribute to the same combination of $n$ share values. In such a case, if the secret image is of little variation in gray-level, e.g. textual images, its content might be leaked from a single shadow.

For Construction 1 of $(2,3)$ threshold access structure, the experimental results on coefficient fields $G F(251)$ and $G F\left(2^{8}\right)$ can be found in Figures 1 and 2 respectively, in which any single shadow reveals the content of the secret image. 


\section{LOIS LOIS LOIS LOIS}

(a)

(b)

(c)

(d)

Fig. 1. Experimental results of Construction 1 on $G F(251)$, (a) the original secret image with image size $300 \times 300$, (b) shadow 1 with image size $150 \times 300$, (c) shadow 2 with image size $150 \times 300$, (d) shadow 3 with image size $150 \times 300$

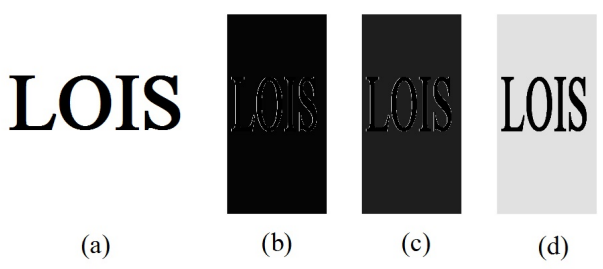

Fig. 2. Experimental results of Construction 1 on $G F\left(2^{8}\right)$, (a) the original secret image with image size $300 \times 300$, (b) shadow 1 with image size $150 \times 300$, (c) shadow 2 with image size $150 \times 300$, (d) shadow 3 with image size $150 \times 300$

Remark: The above security defect seems to be obvious, so why it is not discovered in previous studies? One of the reasons may be that in previous experiments, they only use Construction 1 to encode natural dithered images and never use Construction 1 to encode textual images. But we think a good secret image sharing scheme should be able to deal with all kinds of images, and shouldn't make any restriction on the content of the image.

\section{The Proposed Computational Secure $(k, n)$-TSISS}

In this section, we first propose a new $(k, n)$-TSISS. Then we will prove that this $(k, n)$-TSISS is computational secure.

Definition 2 (Computational secure). Let the secret $s$ be drawn from $G F\left(2^{m}\right)$. A $(k, n)$-SSS is computational secure if for any probability polynomialtime $(P P T)$ algorithm $A, \operatorname{Pr}\left[A\left(s_{i_{1}}, \ldots, s_{i_{k-1}}\right)=s\right]$ is negligible in $m$, which is the success probability of getting the secret $s$ from any $k-1$ shadows $s_{i_{1}}, \ldots, s_{i_{k-1}}$.

Remark: In other words, it is computational infeasible to invert the $(k, n)$ secret sharing scheme from any $k-1$ shadows $s_{i_{1}}, \ldots, s_{i_{k-1}}$.

To achieve computational security, we need to have a computational hardness assumption. For some knowledge of computational security and AES in CBC mode, one can refer to [7, 13]. In this paper, we will use the following assumption: 
Assumption 1. It is computational infeasible to invert AES in CBC mode without the key.

The proposed computational secure $(k, n)$-TSISS, also abbreviated as $(k, n)$ CSTSISS, contains two parts: Construction 2. which is the sharing program run by the dealer in the encoding phase; Construction 3, which is the revealing program run by the shadow holders in the decoding phase.

\section{Construction 2.}

Input: A secret image $S$.

Output: $n$ shadows $\left(S_{1}, K_{1}, I V\right),\left(S_{2}, K_{2}, I V\right), \ldots,\left(S_{n}, K_{n}, I V\right)$.

Step 1. Pick up a random 128 bit key $K$ and a random 128 bit initialization vector $I V$.

Step 2. Encrypt the secret image $S$ by AES with key $K$ and initialization vector $I V$ in $C B C$ mode. Each time we encrypt a 128 bit block, which contains 16 pixels for gray level image. The encrypted secret image is denoted as $D$.

Step 3. Fix $f(x)=p_{1}+p_{2} x+p_{3} x^{2}+\ldots+p_{k} x^{k-1}$, where $p_{1}, p_{2}, \ldots, p_{k}$ are $k$ successive pixels. Then the pixel value for each shadow is calculated as $q_{i}=f(i)$ for $i=1, \ldots, n$. Repeat the above process until all pixels of $D$ have been shared. The shadows are denoted as $S_{1}, S_{2}, \cdots, S_{n}$.

Step 4. Pick up a random $k-1$ degree polynomial $g(x)=a_{1}+a_{2} x+a_{3} x^{2}+$ $a_{4} x^{3}+\ldots+a_{k} x^{k-1}$ with $a_{1}=K$ and $a_{2}, \ldots, a_{k} \stackrel{r}{\in} G F\left(2^{128}\right)$. Then the shadows are calculated by $K_{i}=g(i)$ for $i=1, \ldots, n$.

Step 5. Participant $i$ is distributed $\left(S_{i}, K_{i}, I V\right)$ as his shadow, where $i=1,2$, $\ldots, n$.

Remark: In Step 3., we use the $(k, n)$-VSSS, whose security is not guaranteed, and in Step 4., we use Shamir's $(k, n)$-SSS, which is information theoretic secure. The coefficient field that we use in Step 3. is $G F\left(2^{8}\right)$.

\section{Construction 3.}

Input: Any $k$ shadows: $\left(S_{i_{1}}, K_{i_{1}}, I V\right), \ldots,\left(S_{i_{k}}, K_{i_{k}}, I V\right)$.

Output: $A$ image $S$.

Step 1. Use $K_{i_{1}}, \ldots, K_{i_{k}}$ and Lagrange's interpolation to recover the constant term $K$ of $g(x)$.

Step 2. Use $S_{i_{1}}, \ldots, S_{i_{k}}$ and Lagrange's interpolation to recover all coefficients $p_{1}, p_{2}, \ldots, p_{k}$ of $f(x)$. Repeat the above process until all pixels of $D$ have been recovered.

Step 3. Decrypt $D$ by $A E S$ in $C B C$ mode with key $K$ and initialization vector $I V$. The output is $S$.

Theorem 3. If Assumption 1 holds, then the proposed $(k, n)$-CSTSISS is computational secure.

Due to page limit, the proof of Theorem 3 is omitted in this version. If you are interested in details of the proof, you can refer to the full version on eprint. 
Compared to previous $(k, n)$-TSISSs, the price we have payed out is $2 \times 128 \times n$ bits more storage space in overall, and more time for the $A E S$ encryption and decryption processes.

The experimental results of the proposed $(2,3)$-CSTSISS can be found in Figure 3, in which shadow images (b,c,d) are noise-like and provide no information about the content of the secret image (a). Compared to previous $(k, n)$-TSISSs, the proposed $(k, n)$-CSTSISS indeed does not have security defect for sharing textual images.

\section{LOIS}

(a)

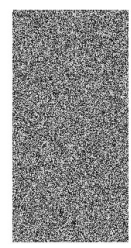

(b)

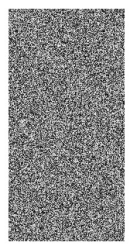

(c)

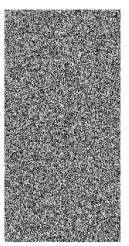

(d)

Fig. 3. Experimental results of the proposed (2,3)-CSTSISS: (a) the original secret image with image size $300 \times 300$, (b) shadow 1 with image size $150 \times 300$, (c) shadow 2 with image size $150 \times 300$, (d) shadow 3 with image size $150 \times 300$

\section{Conclusions}

In this paper, we have shown that the $(k, n)$-VSSS, being extensively used in the studies of $(k, n)$-TSISS, does not have security guarantees. Furthermore, we have found those studies' security defect for sharing textual images and then patched up this security defect by adding an AES encryption process before the sharing process, which combines the beauty of small shadow size with computational security guarantee.

Acknowledgments. This work was supported by NSFC grant No. 60903210, the "Strategic Priority Research Program" of the Chinese Academy of Sciences No. XDA06010701 and the IIE's Projects No. Y1Z0011102, No. Y3Z001B102, No. Y2Z0011102 and No. Y3Z0071C02.

\section{References}

1. Ateniese, G., Blundo, C., Santis, A.D., Stinson, D.: Visual cryptography for general access structures. Information and Computation 129, 86-106 (1996)

2. Chan, C., Sung, P.: Secret image sharing with steganography and authentication using dynamic programming strategy. In: PCSPA, pp. 382-395 (2010)

3. Chang, C., Hsieh, Y., Lin, C.: Sharing secrets in stego images with authentication. Pattern Recognition 41, 3130-3137 (2008) 
4. Cover, T., Thomas, J.: Elements of information theory, 2nd edn. Wiley Interscience, New York (2006)

5. Elsheh, E., Hamza, A.B.: Robust approaches to 3D object secret sharing. In: Campilho, A., Kamel, M. (eds.) ICIAR 2010. LNCS, vol. 6111, pp. 326-335. Springer, Heidelberg (2010)

6. Goldreich, O.: Randomized methods in computation (2001), http://www.wisdom.weizmann.ac.il/ oded/rnd.html

7. Goldwasser, S., Bellare, M.: Lecture notes on cryptography (2008), http://cseweb.ucsd.edu/ mihir/papers/gb.pdf

8. Guo, T., Liu, F., Wu, C.: Multi-pixel encryption visual cryptography. In: Wu, C.K., Yung, M., Lin, D. (eds.) Inscrypt 2011. LNCS, vol. 7537, pp. 86-92. Springer, Heidelberg (2012)

9. Guo, T., Liu, F., Wu, C.: On the equivalence of two definitions of visual cryptography scheme. In: Ryan, M.D., Smyth, B., Wang, G. (eds.) ISPEC 2012. LNCS, vol. 7232, pp. 217-227. Springer, Heidelberg (2012)

10. Guo, T., Liu, F., Wu, C.: Threshold visual secret sharing by random grids with improved contrast. The Journal of Systems and Software 86, 2094-2109 (2013)

11. Guo, T., Liu, F., Wu, C.: $\mathrm{k}$ out of $\mathrm{k}$ extended visual cryptography scheme by random grids. Signal Processing 94, 90-101 (2014)

12. Huang, C., Hsieh, C., Huang, P.: Progressive sharing for a secret image. The Journal of Systems and Software 83, 517-527 (2010)

13. Katz, J., Lindell, Y.: Introduction to modern cryptography. CRC Press (2007)

14. Li, P., Ma, P., Su, X., Yang, C.: Improvements of a two-in-one image secret sharing scheme based on gray mixing model. Journal of Visual Communication and Image Representation 23, 441-453 (2012)

15. Liu, F., Guo, T., Wu, C., Qian, L.: Improving the visual quality of size invariant visual cryptography scheme. Journal of Visual Communication and Image Representation 23, 331-342 (2012)

16. Naor, M., Shamir, A.: Visual cryptography. In: De Santis, A. (ed.) EUROCRYPT 1994. LNCS, vol. 950, pp. 1-12. Springer, Heidelberg (1995)

17. Shamir, A.: How to share a secret. Communications of the ACM 22(11), 612-613 (1979)

18. Thien, C., Lin, J.: Secret image sharing. Computers and Graphics 26, 765-770 (2002)

19. Thien, C., Lin, J.: An image-sharing method with user-friendly shadow images. IEEE Transactions on Circuits and Systems for Video Technology 13, 1161-1169 (2003)

20. Ulutas, M., Ulutas, G., Nabiyev, V.: Medical image security and epr hiding using shamir's secret sharing scheme. The Journal of Systems and Software 84, 341-353 (2011)

21. Wang, R., Chien, Y., Lin, Y.: Scalable user-friendly image sharing. Journal of Visual Communication and Image Representation 21, 751-761 (2010)

22. Wang, R., Shyu, S.: Scalable secret image sharing. Signal Processing: Image Communication 22, 363-373 (2007)

23. Yang, C., Chen, T., Yu, K., Wang, C.: Improvements of image sharing with steganography and authentication. The Journal of Systems and Software 80, 1070-1076 (2007) 
24. Yang, C., Ciou, C.: A comment on "sharing secrets in stegoimages with authentication". Pattern Recognition 42, 1615-1619 (2009)

25. Yang, C., Ciou, C.: Image secret sharing method with two-decoding-options: Lossless recovery and previewing capability. Image and Vision Computing 28, 1600-1610 (2010)

26. Yang, C., Huang, Y., Syue, J.: Reversible secret image sharing based on shamir's scheme with discrete haar wavelet transform. In: ICECE, vol. 16-18, pp. 1250-1253 (2011)

27. Yang, C., Ouyang, J., Harn, L.: Steganography and authentication in image sharing without parity bits. Optics Communications 285, 1725-1735 (2012)

28. Zhao, R., Zhao, J., Dai, F., Zhao, F.: A new image secret sharing scheme to identify cheaters. Computer Standards and Interfaces 31, 252-257 (2009) 